\title{
Interferon regulatory factor-1 regulates cisplatin-induced apoptosis and autophagy in A549 lung cancer cells
}

\author{
Lemeng Zhang ${ }^{1}$ (i) $\cdot$ Tianli Cheng $^{1} \cdot$ Hua Yang ${ }^{1} \cdot$ Jianhua Chen ${ }^{1} \cdot$ Xiaoping Wen $^{1} \cdot$ Zhou Jiang $^{1} \cdot$ Huihuang Yi ${ }^{1}$. \\ Yongzhong Luo ${ }^{1}$ (i)
}

Received: 20 August 2021 / Accepted: 23 December 2021 / Published online: 29 January 2022

(c) The Author(s) 2022

\begin{abstract}
This study aimed to investigate the expression and function of interferon regulatory factor-1 (IRF-1) in non-small cell lung cancer (NSCLC). IRF-1 expression and its prognostic value were investigated through bioinformatic analysis. The protein expression levels of IRF-1, cleaved caspase 3, and LC3-I/II were analyzed by western blotting. A lentiviral vector was used to overexpress or knockdown IRF-1 in vitro. Mitochondrial membrane potential (MMP) and reactive oxygen species (ROS) were analyzed by JC-1 and DCFH-DA staining, respectively. ATP, SOD, MDA, cell viability, LDH release, and caspase 3 activity were evaluated using commercial kits. Compared to the levels in normal tissues, IRF-1 expression was significantly lower in lung cancer tissues and was a prognostic factor for NSCLC. Cisplatin treatment-induced IRF-1 activation, ROS production, ATP depletion, SOD consumption, and MDA accumulation in A549 lung cancer cells. IRF-1 overexpression promoted mitochondrial depolarization, oxidative stress, and apoptotic cell death and inhibited autophagy in A549 cells, and these effects could be reversed by IRF-1 knockdown. These data suggest that IRF-1 regulates apoptosis, autophagy and oxidative stress, which might be served as a potential target for increasing chemotherapy sensitivity of lung cancer.
\end{abstract}

Keywords NSCLC $\cdot$ Cisplatin $\cdot$ Apoptosis $\cdot$ Autophagy $\cdot$ IRF-1

\section{Background}

Lung cancer is a malignant disease with the highest morbidity and mortality worldwide, as it accounts for $18 \%$ of cancer-associated deaths [1]. Approximately $80 \%$ of lung cancers are non-small cell lung cancer (NSCLC) [2]. Although significant progress has been made in targeted therapy and immunotherapy for NSCLC [3, 4], the prognosis remains poor, and the 5-year survival rate of patients with advanced disease is less than 15\% [5].

Cisplatin is the first-line chemotherapy drug for NSCLC [6]. Re-sensitizing resistant tumors to cisplatin remains a major challenge in clinical treatment. The main mechanism of cisplatin-induced cell death is apoptosis [7, 8], and inhibitors of apoptosis proteins drive cisplatin resistance [9]. Autophagy is also involved in cisplatin resistance [10], and

Yongzhong Luo

luoyongzhong@hnca.org.cn

1 Thoracic Medicine Department 1, Hunan Cancer Hospital, Tongzipo Rd 283\#, Yuelu District, Changsha 410013, Hunan Province, People's Republic of China it has been suggested that activation of autophagy promotes cisplatin resistance in ovarian cancer [11]. In renal cell carcinoma cells, mitochondrial depolarization and reactive oxygen species (ROS) production were shown to be involved in resistance to chemotherapy [12], sorafenib-induced depolarization, and ROS accumulation, which in turn activates caspase-8, an important regulator of apoptosis. Han et al. reported that mitochondrial fission-induced cisplatin resistance in hypoxic ovarian cancer cells through ROS, while inhibition of mitochondrial fission increased cisplatin sensitivity [13].

Interferon regulatory factor-1 (IRF-1) is a nuclear transcription factor that plays key roles in interferon expression, lymphocyte growth and differentiation, innate and acquired immunity, and other immune-related events $[14,15]$. Recent studies have implicated IRF-1 in the pathogenesis of malignant diseases. Deletions or mutations in IRF-I have been found in numerous human tumor tissues, including myelodysplastic syndrome [14], leukemia [16], gastric cancer [17] and breast cancer. In addition, IRF-1 mediates apoptosis in ovarian cancer [18] and breast cancer [19] and regulates autophagy in breast cancer cells [20], splenocytes [21] and 
macrophages [22], which are cell fate determinants. Interestingly, IRF-1 could reverse the multiple drug resistance of gastric cancer by decreasing the expression of P-glycoprotein [23], and high expression of IRF-1 contributes to 5 -fluorouracil chemosensitivity in gastric cancer cells [24]. Nevertheless, the activation status and function of IRF-1 in lung cancer and cisplatin resistance have not been reported.

In the current study, we first explored the expression of IRF-1 in lung cancer tissues using public databases and clinical samples. Next, we observed cisplatin-induced IRF-1 activation and explored its effects on mitochondrial homeostasis, autophagy, and apoptosis in lung cancer cells. A comprehensive, in-depth understanding of the activity and mechanism of IRF-1 in NSCLC is of theoretical and practical significance for the treatment of lung cancer.

\section{Methods}

\section{Analysis of IRF-1 expression in silico}

GEPIA [25] (http://gepia.cancer-pku.cn/index.html) was utilized to explore the expression of IRF-1 in different tumors in the cancer genome atlas (TCGA) database. Scatter plots and histograms were drawn, and survival analyses were conducted. Expression data and corresponding clinical information for lung adenocarcinoma (LUAD) and lung squamous cell carcinoma (LUSC) in TCGA database were acquired using UCSC Xena (http://xena.ucsc.edu/) [26]. The original count values were converted to transcripts per million (TPM) using TPM standardization. The extracted IRF-1 values were used for further analysis. IRF-1 protein expression levels in LUAD and LUSC were verified using the Human Protein Atlas (HPA) [27], which is designed for the investigation of protein expression in various human tissues and organs.

\section{Reagents and antibodies}

Cisplatin, methyl thiazolyl tetrazolium (MTT), and LDHdependent cytotoxic nonradioactive cytotoxicity assays were obtained from Promega (Madison, WI, USA). The LC3B antibody was purchased from Novus Biologicals (Littleton, CO, USA). $N$-acetylcysteine (NAC), $\beta$-actin, and 2,7-dichloro-fluorescin diacetate (DCFH-DA) were purchased from Sigma-Aldrich (St. Louis, MO, USA). An anti-IRF-1 antibody was obtained from Santa Cruz Biotechnology (Santa Cruz, CA, USA). The Caspase 3 Fluorometric Assay kit was obtained from BioVision (Milpitas, CA, USA). Goat anti-rabbit secondary antibodies were purchased from Thermo Fisher Scientific Inc. (Waltham, MA, USA). 5, 5',6,6'-Tetrachloro-1,1',3,3'-tetraethylbenzimidazolylcarbocyanine iodide (JC-1) was obtained from Invitrogen (Carlsbad, CA, USA). Assay kits for ATP, superoxide dismutase (SOD), and malondialdehyde (MDA) were obtained from Nanjing Jiancheng Bioengineering Institute. (Nanjing, China). The FITC-Annexin V/7-AAD apoptosis detection kit was obtained from BD Biosciences (USA).

\section{Clinical sample collection}

This study included lung cancer patients who underwent surgery at Hunan Cancer Hospital (Changsha, Hunan, China) between August 2014 and August 2015. All diagnoses were confirmed from histopathological reports. The Ethics Committee of Hunan Cancer Hospital approved this study, and informed consent was obtained from all study patients.

\section{Cell culture}

The human lung cancer cell lines A549, SK-MES-1, H1299, H460, H358, and H1975 were obtained from American Type Culture Collection (Manassas, VA, USA). All cells were cultured in PRMI-1640 or DMEM supplemented with $10 \%$ FBS (Biowest, South America Origin), $100 \mathrm{U} / \mathrm{ml}$ penicillin sodium, and $100 \mathrm{mg} / \mathrm{mL}$ streptomycin sulfate at $37{ }^{\circ} \mathrm{C}$ in a humidified atmosphere containing $5 \% \mathrm{CO}_{2}$, as previously described [28].

\section{Cell transfection}

IRF-1 shRNA and overexpression lentiviral vectors (Hanyin, Shanghai, China) were prepared at a titer of $1 \times 10^{9} \mathrm{TU} /$ $\mathrm{mL}$. These vectors were transfected into cells using $5 \mu \mathrm{g} / \mathrm{mL}$ polyamine in RMI-1640 medium at a multiplicity of infection (MOI) of 20:1. After $4 \mathrm{~h}$ of transfection, the cells were cultured for $48 \mathrm{~h}$ in fresh culture medium. The efficiency of IRF-1 knockdown and overexpression was assessed by western blotting.

\section{Western blotting}

Following treatment with cisplatin $(20 \mu \mathrm{M})$, cytoplasmic and nuclear proteins were extracted according to the manufacturer's instructions. Then, the proteins were separated by electrophoresis and transferred to Trans-Blot nitrocellulose membranes (Bio-Rad Laboratories). After blocking with 5\% nonfat milk for $1 \mathrm{~h}$ at room temperature and incubation with the primary antibodies and the HRP-conjugated secondary antibody, the color reaction was initiated using the Super Signal West Pico chemiluminescent kit (Pierce Chemical Co.), and the blot was exposed to film (Eastman Kodak). The data were analyzed with densitometry using Image $\mathbf{J}$ software (Bethesda, MD, USA). Histone was used as an internal loading control. 


\section{Mitochondrial membrane potential and reactive oxygen species detection}

As described in a previous study [14], mitochondrial membrane potential was measured using JC-1 staining, and ROS was detected using DCFH-DA staining.

\section{Detection of intracellular ATP, SOD, and MDA}

Intracellular ATP, MDA, and SOD levels were detected using corresponding assay kits according to the manufacturer's instructions.

\section{Cell viability detection and lactate dehydrogenase release}

The MTT assay was conducted to measure cell viability, and lactate dehydrogenase (LDH) was determined using the LDHdependent Cytotoxic Non-Radioactive Cytotoxicity Assay kit, as described by Chen et al. [29].

\section{Caspase 3 activity assay and apoptotic cell death detection by flow cytometry}

Caspase 3 activity was measured using the Caspase 3 Fluorometric Assay kit according to the manufacturer's instructions. Apoptotic cells were detected using the FITC-Annexin V/7AAD apoptosis detection kit according to the manufacturer's instructions.

\section{Statistical analysis}

Data were processed using SPSS software (version 23.0). Quantitative data are shown as the mean \pm standard deviation of three replicates as determined with Student's t-test or one-way analysis of variance (ANOVA) between two or more groups. Repeated measures ANOVA followed by an LSD test was used to analyze differences within groups. Samples from TCGA were categorized into high- and low-expression groups based on the upper and lower quartiles of IFR-1 expression. Kaplan-Meier curves and log-rank tests were used to analyze the correlation between IRF-1 expression and patient survival. Clinical phenotypes between groups were compared using the Chi-square test. Univariate Cox regression analysis was used to screen survival-related factors. A P value less than 0.05 was considered statistically significant.

\section{Results}

\section{IRF-1 is depleted in non-small cell lung cancer}

We used GEPIA for a pan-tumor analysis of IRF-1 expression, which showed that IRF-1 was differentially expressed in cancer tissues and its expression was inconsistent in different cancer tissues. IRF-1 expression was downregulated in adrenocortical carcinoma (ACC), kidney chromophobe $(\mathrm{KICH})$, LUAD, and LUSC (Fig. 1A , B). Of particular note, IRF-1 expression was downregulated in LUAD and LUSC samples compared to the expression in normal samples (Fig. 1C). Analysis of the HPA database further verified the low IRF-1 protein levels in LUAD and LUSC, and the results indicated that IRF-1 protein levels were decreased in lung cancer tissues and lymph nodes compared to the levels in normal lymph nodes (Fig. 1D). In addition, western blot analysis of IRF-1 in clinical lung cancer tissues showed that IRF-1 expression was significantly downregulated in cancer tissues compared to the levels in normal tissues (Fig. 1E). Therefore, we concluded that IRF-1 was depleted in NSCLC tissues.

\section{IRF-1 is a prognostic factor}

We further investigated the clinical value of IRF-1 in NSCLC using clinical data from TCGA. Table 1 shows the clinical information of patients obtained from TCGA. Based on the upper and lower quartiles of IFR-1 expression, the samples were grouped into high- and low-expression groups. There was no significant difference in the clinical characteristics between the high and low IRF-1 groups. Survival analysis indicated that IRF-1 expression was significantly correlated with prognosis $(P<0.05)$ (Fig. 1F). Univariate Cox regression analysis showed that the response to chemotherapy ( $\mathrm{HR}=0.233, P=0.009)$, metastasis $(\mathrm{HR}=2.129$, $P<0.001)$, lymph node status ( $\mathrm{HR}=2.637, P<0.001)$, and tumor stage $(\mathrm{HR}=2.278, P<0.001)$ had significant impacts on patient prognosis. In addition to these clinical factors, IRF-1 was also associated with patient prognosis, with an HR of 1.003 (95\% CI 1.000-1.005, $P=0.031$ ) (Table 2).

\section{Cisplatin induces IRF-1 activation in vitro}

Cisplatin-induced IRF-1 activation was assessed in different lung cancer cell lines by Western blotting. As shown in Fig. 2A, cisplatin-induced IRF-1 activation differently in the tested cell lines; cisplatin-induced IRF-1 activation only in A549, SK-MES-1, and H460 cells, but not in the other tested lung cancer cells. Additionally, cisplatin-induced IRF-1 activation in A549 cells reached a peak at $8 \mathrm{~h}$ and returned to 
A

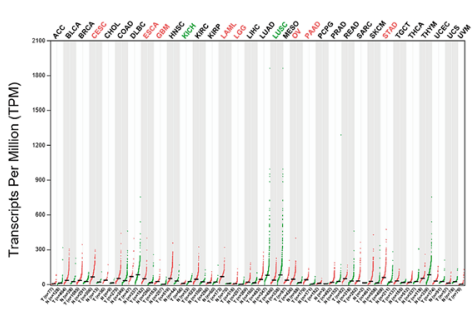

B

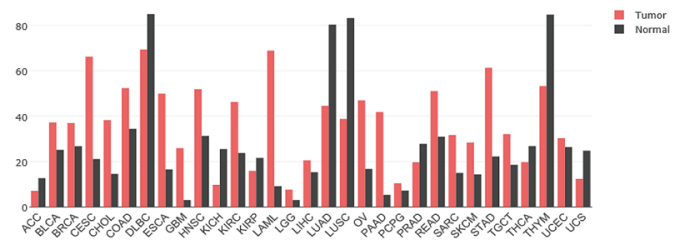

C

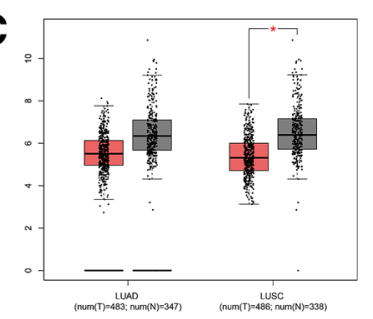

D

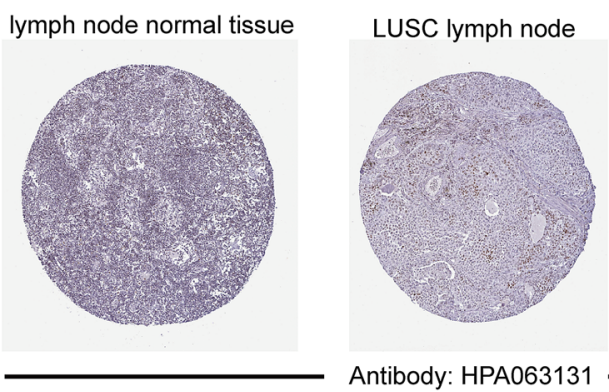

LUAD lymph node
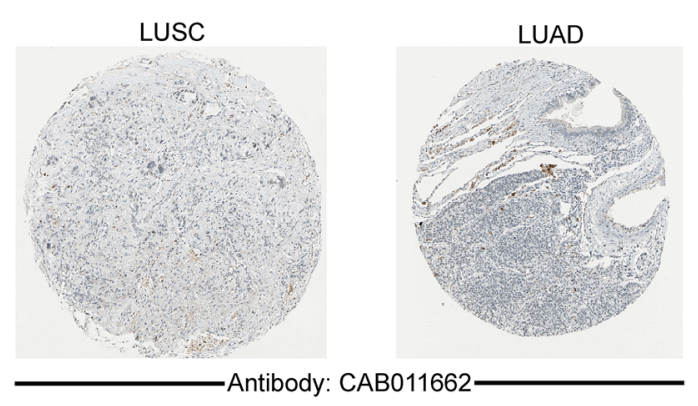

$\mathbf{E}$

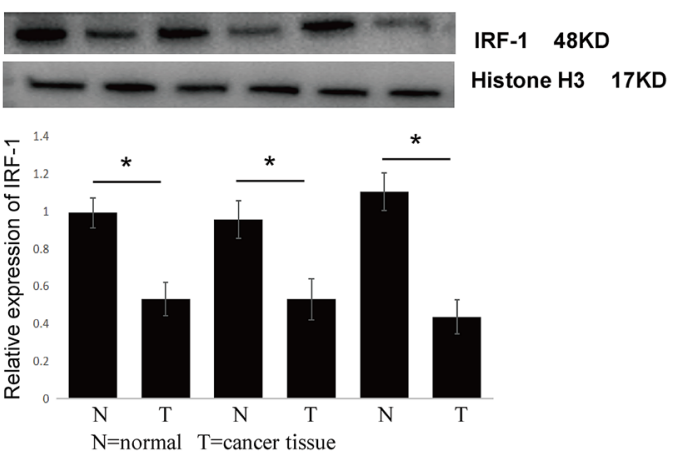

$\mathbf{F}$

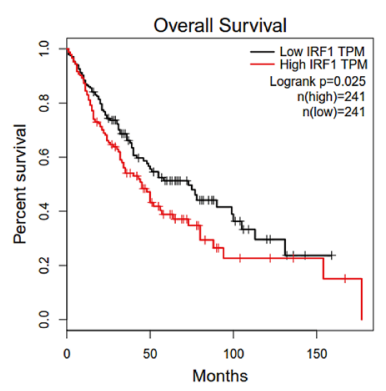

Fig. 1 IRF-1 expression in lung cancer: TCGA database, clinical samples, and A549 cells. A Scatter plot showing pan-tumor IRF-1 expression profiles with paired normal tissues as analyzed by GEPIA. Each point represents the IRF-1 expression in one sample; B Bar plot showing the pan-tumor IRF-1 expression profiles with paired normal tissues as analyzed by GEPIA. The height of the columns represents the median IRF-2 expression in tumor and normal tissues; C Boxplot showing decreased IRF-1 expression levels in LUAD and LUSC sam-

ples compared to the normal controls; D IRF-1 immunohistochemical staining data from the HPA database; E, IRF-1 protein expression in NSCLC clinical samples as determined by western blotting; $\mathbf{F}$ Kaplan-Meier survival curve showing the prognostic value of IRF-1 expression in NSCLC in a TCGA dataset; IRF-1 protein expression in A549 cells after cisplatin $(20 \mu \mathrm{M})$ treatment for $8 \mathrm{~h}$ as determined by western blotting

baseline at $24 \mathrm{~h}$ after cisplatin stimulation (Fig. 2B), demonstrating that IRF-1 levels were elevated by cisplatin in a time-dependent manner. Since the A549 cell line showed the highest levels of IRF-1 transcription and expression following cisplatin treatment, it was chosen for further study.

\section{Cisplatin induces ROS production and mitochondrial depolarization}

Compared with control cells, cisplatin-treated A549 cells showed lower mitochondrial membrane potential (Fig. 3A) and increased ROS production (Fig. 3B), suggesting that cisplatin treatment leads to mitochondrial depolarization and oxidative stress in A549 cells. We next determined the effects of cisplatin on intracellular ATP levels and oxidative

stress-related biochemical indices, i.e., SOD and MDA levels. As shown in Fig. 3C-E, cisplatin treatment significantly promoted ATP depletion in A549 cells in a time-dependent manner. Similarly, cisplatin treatment significantly increased SOD consumption and MDA accumulation in A549 cells in a time-dependent manner.

\section{IRF-1 overexpression promotes mitochondrial depolarization and oxidative stress}

To study the effect of IRF-1 on mitochondrial depolarization and oxidative stress in A549 cells, we overexpressed IRF-1. Western blotting showed that IRF-1 was upregulated after transfection with an IRF-1 overexpression lentiviral vector (Fig. 4A). IRF-1 overexpression disrupted mitochondrial 
Table 1 Correlation between IRF-1 expression and clinical parameters

\begin{tabular}{|c|c|c|c|c|}
\hline \multirow[t]{2}{*}{ Clinical features } & \multirow[t]{2}{*}{$N$} & \multicolumn{2}{|l|}{ IRF-1 } & \multirow[t]{2}{*}{$P$ value } \\
\hline & & Low expression & $\begin{array}{l}\text { High } \\
\text { expres- } \\
\text { sion }\end{array}$ & \\
\hline \multicolumn{5}{|l|}{ Age (years) } \\
\hline$<=65$ & 188 & 136 & 52 & 0.344 \\
\hline$>65$ & 299 & 229 & 70 & \\
\hline \multicolumn{5}{|l|}{ Gender } \\
\hline Female & 129 & 92 & 37 & 0.315 \\
\hline Male & 367 & 280 & 87 & \\
\hline \multicolumn{5}{|l|}{ Metastasis } \\
\hline M0 & 407 & 303 & 104 & 0.2 \\
\hline M1 & 7 & 7 & 0 & \\
\hline \multicolumn{5}{|c|}{ Lymph node status } \\
\hline No & 316 & 240 & 76 & 0.563 \\
\hline $\mathrm{N} 1+2+3$ & 175 & 128 & 47 & \\
\hline \multicolumn{5}{|l|}{ Tumor stage } \\
\hline $\mathrm{T} 1+\mathrm{T} 2$ & 403 & 303 & 100 & 0.947 \\
\hline $\mathrm{T} 3+\mathrm{T} 4$ & 93 & 69 & 24 & \\
\hline
\end{tabular}

Table 2 Cox regression analysis of prognostic factors

\begin{tabular}{lllc}
\hline & HR & $95 \%$ CI & $P$ value \\
\hline Age (years) & 1.221 & $0.907-1.642$ & 0.188 \\
Gender & 1.048 & $0.782-1.406$ & 0.752 \\
IRF-1 expression & 1.003 & $1.000-1.005$ & $0.031^{*}$ \\
Chemotherapy response & 0.233 & $0.078-0.698$ & $0.009 *$ \\
Metastasis & 2.129 & $1.243-3.648$ & $<0.001^{*}$ \\
Lymph node status & 2.637 & $1.957-3.553$ & $<0.001^{*}$ \\
Tumor stage & 2.278 & $1.547-3.353$ & $<0.001^{*}$ \\
\hline
\end{tabular}

$* P$ value $<0.05$ was considered as a significant difference

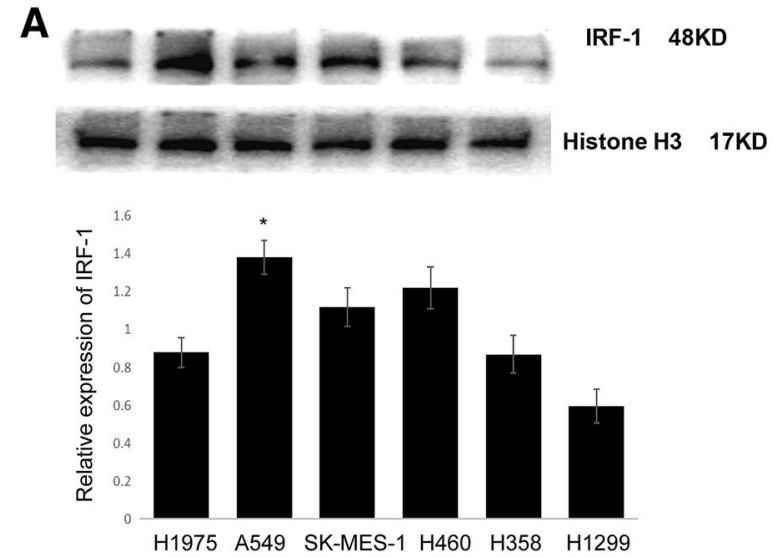

homeostasis and oxidative stress, as evidenced by increased ROS production and upregulated mitochondrial depolarization compared with the control (Fig. 4B, C). IRF-1 overexpression also promoted apoptosis (Fig. 4D) and led to ATP depletion, SOD consumption, and MDA accumulation in A549 cells (Fig. 4E-G).

\section{IRF-1 overexpression promotes apoptotic cell death and inhibits autophagy}

We further investigated the effects of IRF-1 overexpression in A549 cells and found that it led to reduced conversion rate of LC3B-I/II in A549 cells (Fig. 5A, B), indicating an inhibitory effect of IRF-1 on autophagy. In addition, overexpression of IRF-1 increased cleaved-caspase 3 levels (Fig. 5A, C) and increased caspase 3 activity (Fig. 5D), indicating that IRF-1 overexpression promoted apoptosis. Overexpression of IRF-1 also increased LDH release (Fig. 5E) and decreased cell viability (Fig. 5F) in A549 cells. Interestingly, treatment with $5 \mathrm{mM} \mathrm{N}$-acetylcysteine (NAC) alleviated IRF-1-mediated apoptosis and inhibition of autophagy (Fig. 5A-F). Taken together, these results suggest that IRF-1 might enhance the effect of cisplatin on A549 cells by inducing apoptosis, partially through an ROS-dependent pathway.

\section{Knockdown of IRF-1 protects against cisplatin-induced mitochondrial depolarization and oxidative stress}

The expression of IRF-1 was significantly upregulated in cisplatin-treated A549 cells. Transfection of A549 cells with IRF-1 shRNA decreased the expression of IRF-1 (Fig. 6A, B). We then studied the effect of IRF-1 knockdown on cisplatin-induced mitochondrial depolarization and oxidative stress in A549 cells by measuring ATP, MDA, and SOD
B

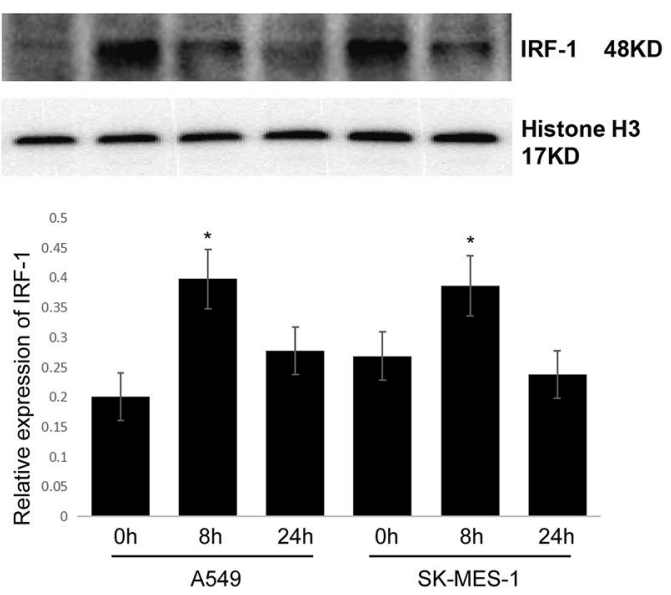

Fig. 2 Cisplatin induces IRF-1 activation in vitro. A IRF-1 protein expression in different lung cancer cell lines after cisplatin ( $20 \mu \mathrm{M})$ treatment as determined by Western blotting; B IRF-1 protein expression in A549 and SK-MES-1 cells at 0, 8, and $24 \mathrm{~h}$ after cisplatin (20 $\mu \mathrm{M})$ treatment 
A
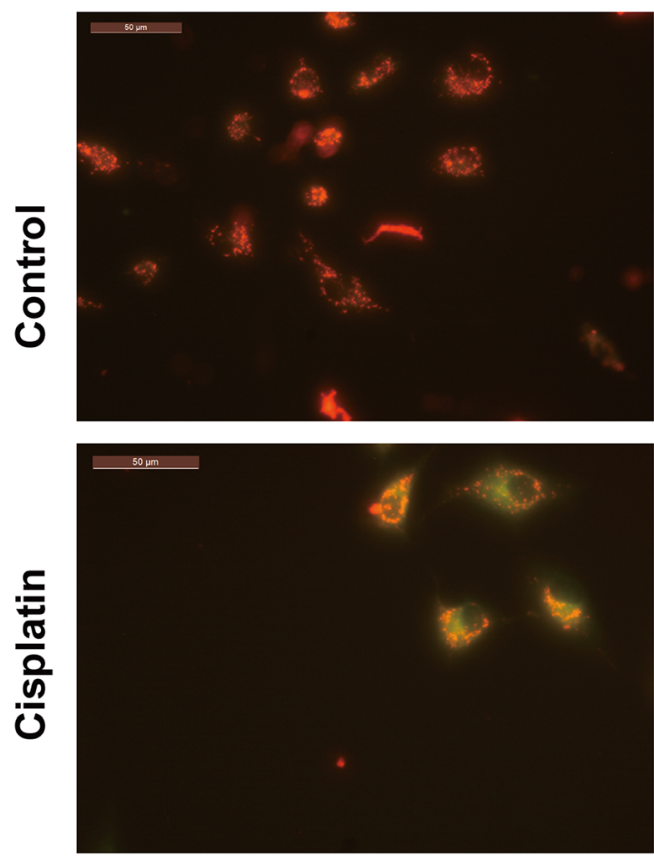

C

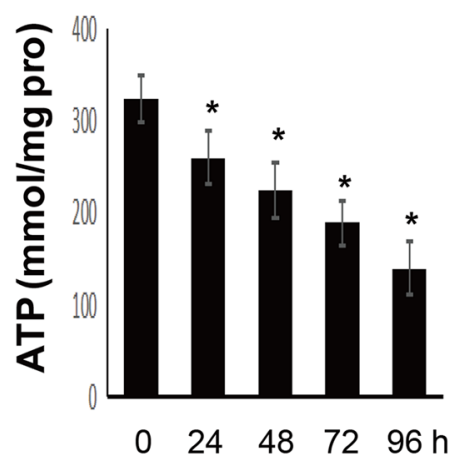

D

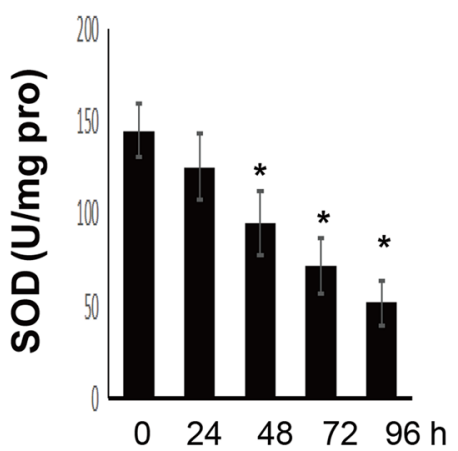

B
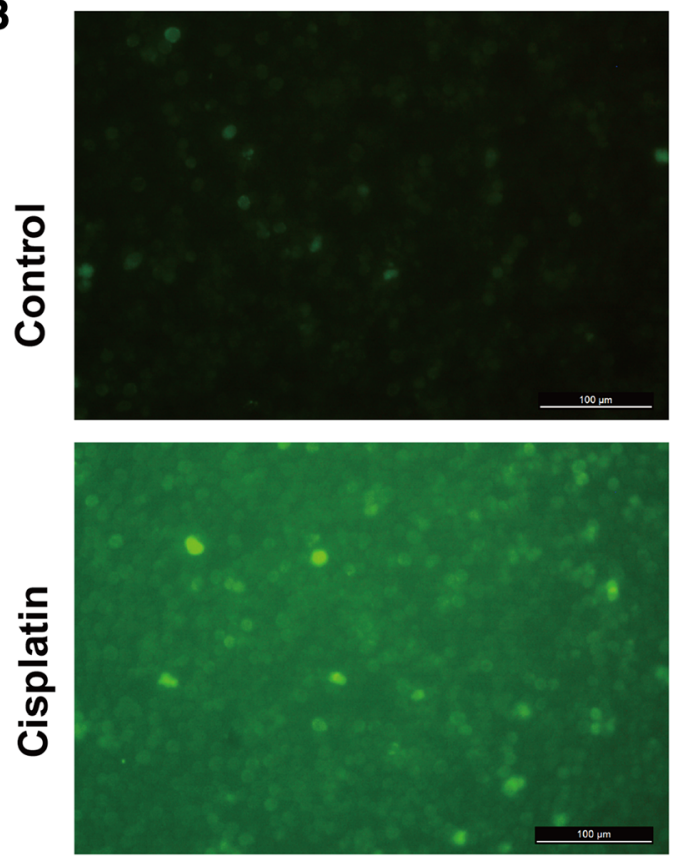

$\mathbf{E}$

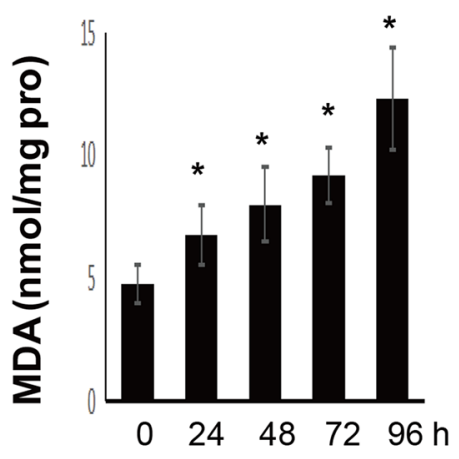

Fig. 3 Cisplatin treatment leads to mitochondrial depolarization and oxidative stress. A Mitochondrial membrane potential as determined by JC-1 staining; B ROS detection by DCFH-DA staining in A549

levels. The results showed that IRF-1 knockdown significantly reduced cisplatin-induced ATP consumption (Fig. 6C) and MDA accumulation (Fig. 6E), and although the difference was not statistically significant, SOD consumption showed a improvement trend (Fig. 6D) following IRF-1 knockdown.

\section{Knockdown of IRF-1 reduces cisplatin-induced apoptosis and promotes autophagy}

Cisplatin-induced apoptosis and autophagy in A549 cells, as demonstrated by increased caspase 3 cleavage and decreased LC3B-I/II conversion (Fig. 7A-C). Transfection with IRF-1 shRNA decreased cleaved caspase 3 levels and increased LC3B-I/II conversion compared with the levels in NC shRNA cells treated with $20 \mu \mathrm{M}$ cisplatin for $8 \mathrm{~h}$, and the levels of ATP (C), SOD (D), and MDA (E) in A549 cells treated with $20 \mu \mathrm{M}$ cisplatin at different time points. $* P<0.05$ vs. the control

transfected cells (Fig. 7A-C), indicating that knockdown of IRF-1 inhibited cisplatin-induced apoptosis and promoted autophagy. Cisplatin treatment increased caspase 3 activity (Fig. 7D) and LDH release (Fig. 7E) and decreased cell viability (Fig. 7F). In IRF-1 knockdown A549 cells, cisplatininduced caspase 3 activity (Fig. 7D) and LDH release were decreased (Fig. 7E) and cell viability was increased (Fig. 7F) compared with the control group. Therefore, knockdown of IRF-1 reduced cisplatin-induced apoptotic cell death and promoted autophagy. 
A

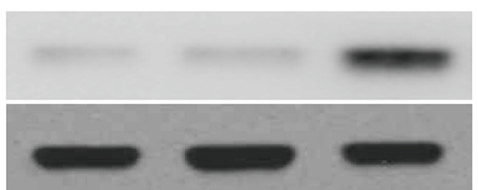

Histone H3 17KD

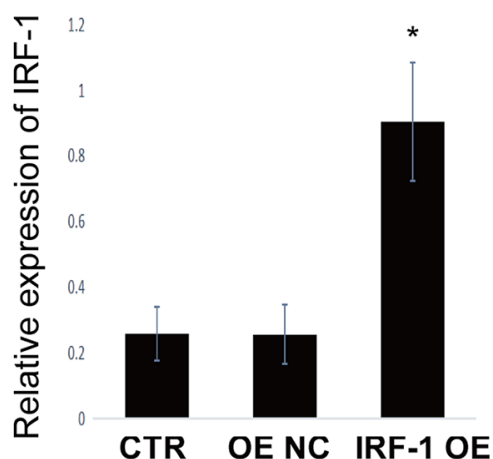

B

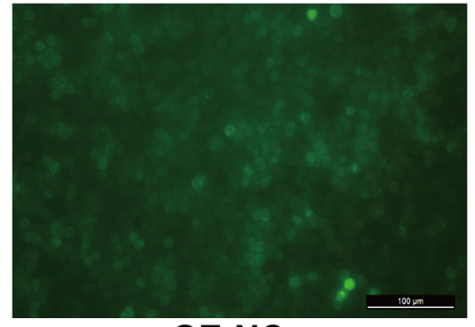

OE NC

C

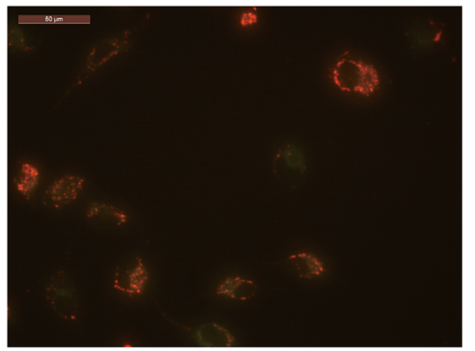

OE NC

D

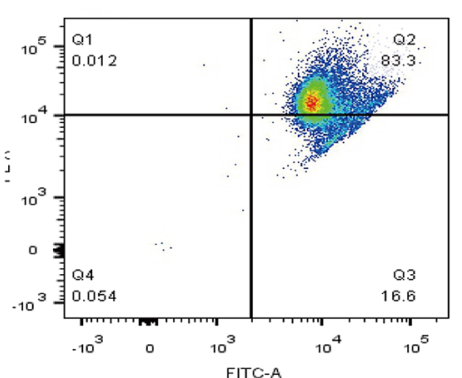

OE NC

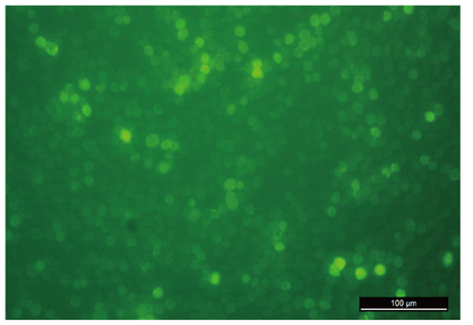

IRF-1 OE

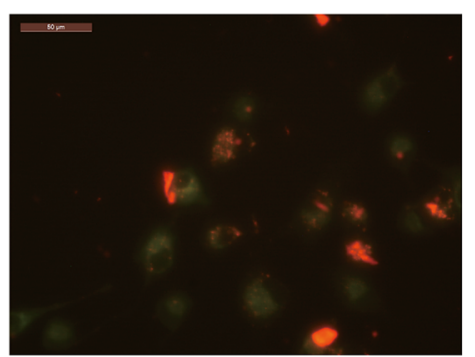

IRF-1 OE

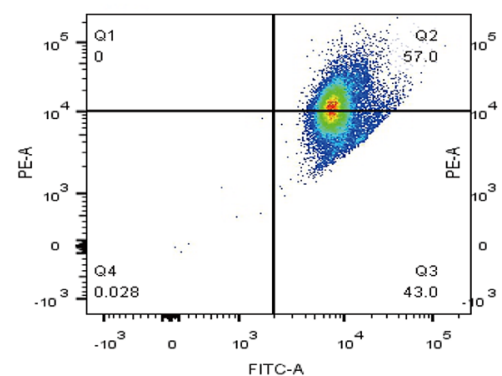

IRF-1 OE
E

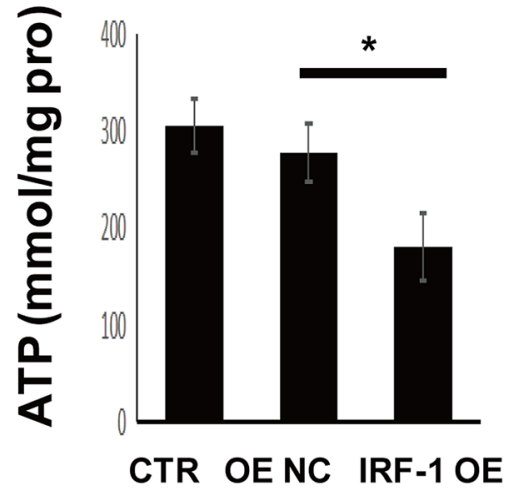

F

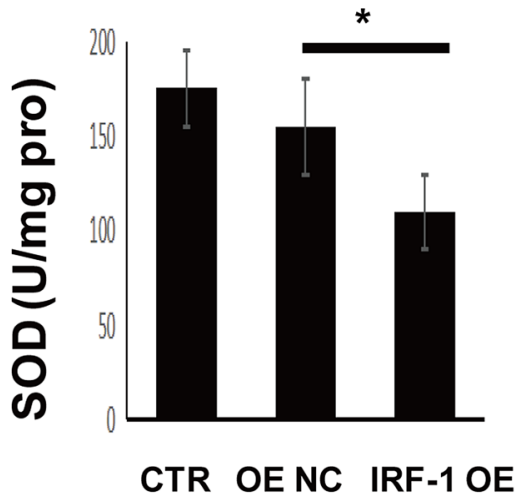

G

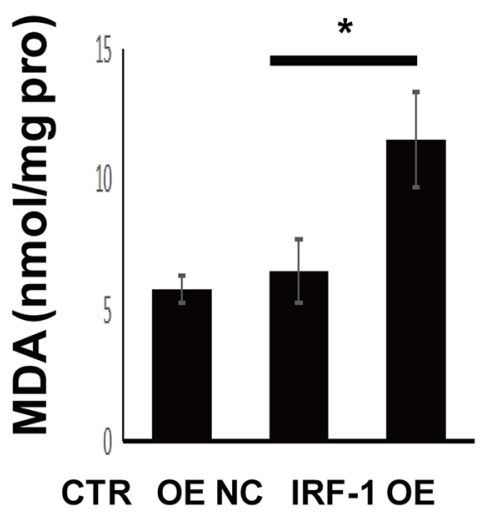

Fig. 4 IRF-1 overexpression promotes mitochondrial depolarization and oxidative stress. A IRF-1 protein expression as determined by western blotting; B ROS release as determined by DCFH-DA staining; C mitochondrial membrane potential as determined by JC-1 staining; D apoptotic cell death as detected by flow cytometry, and
ATP $(\mathbf{E})$, SOD $(\mathbf{F})$, and MDA $(\mathbf{G})$ levels as determined with respective test kits. ${ }^{*} P<0.05$ vs. the control. CTR, control; NC, negative control; OE, overexpression; OE NC, negative control of overexpression group, using empty vector 
A

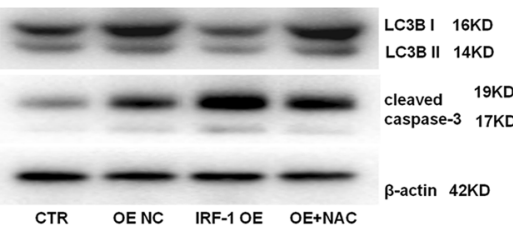

D

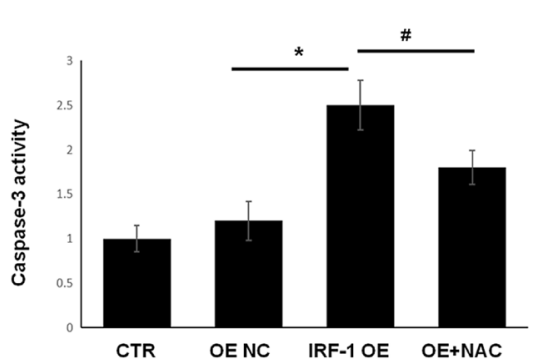

B

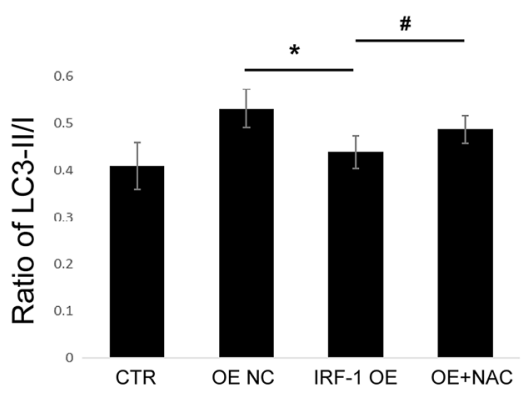

E

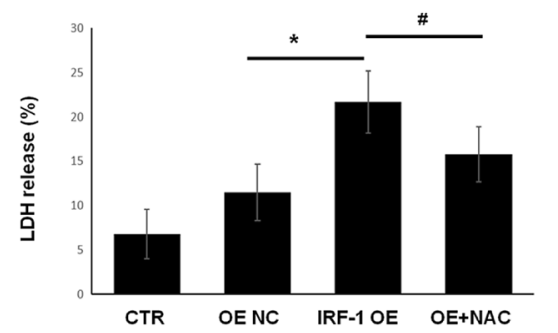

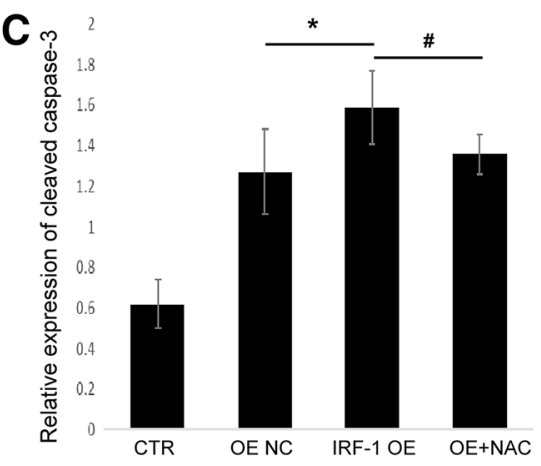

$\mathbf{F}$

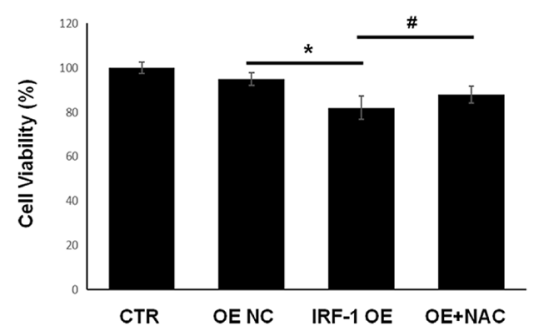

Fig. 5 IRF-1 overexpression promotes apoptosis and inhibits autophagy. A-C Western blotting protein bands and corresponding quantitative analysis show the expression of cleaved caspase 3 and LC3-I/II; D Caspase 3 activity as determined by Caspase 3 Activity

\section{Discussion}

Studies have indicated that deletion or dysfunction of IRF-1 can induce tumor occurrence or progression in various cancers, including gastric cancer [17], breast cancer [30] and hepatocellular carcinoma [31]. IRF-1 was significantly lower in hepatocellular carcinoma tumors than in noncancerous tissues, and activation of IRF- 1 by IFN- $\gamma$-induced PD-L1 expression in vitro [31]. IRF-1 has been implicated in the response of cancer cells to cisplatin. Pavan et al. showed that IRF-1 expression in ovarian cancer cells was significantly increased by cisplatin treatment and IRF-1 overexpression inhibited the transformed phenotype of tumor cells [32]. Yuan et al. reported that IRF-1 was depleted in gastric cancer and that multiple drug resistance could be reversed by activating IRF-1 expression using doxycycline [23]. Consistently, we found that IRF-1 was depleted in NSCLC and cisplatin-induced IRF-1 activation in A549 cells.

Cisplatin-induced apoptosis is important for its antitumor activity. Mitochondria regulate multiple cellular functions, including cellular energy conversion, metabolism, ROS production and the intrinsic apoptotic pathway [33, 34]. Disturbances in mitochondrial homeostasis are important for triggering apoptosis [35]. Previous studies have shown that oxidative stress is a mediator of cisplatin-induced apoptotic cell death and is closely related to chemoresistance [36]. In
Assay Kit; E LDH detection using LDH release reagent; F Cell viability as determined by MTT assay. ${ }^{*} P<0.05$ vs. OE NC, $\# P<0.05$ vs. NAC pretreatment. CTR, control; NC, negative control; OE, overexpression; NAC, $N$-acetylcysteine

this study, we found that overexpression of IRF-1 promoted apoptotic cell death and induced mitochondrial depolarization and oxidative stress in A549 lung cancer cells. Our previous study revealed that cisplatin-induced apoptotic cell death and the autophagic response in A549 cells, but the upstream regulator was unknown [29]. In this study, we demonstrated IRF-1 activation as a possible upstream regulator and showed that an imbalance in mitochondrial homeostasis is the central cisplatin resistance mechanism.

IRF-1 has been implicated in the upregulation of stressinduced apoptosis and downregulation of invasive activity in different cancer cells [37]. IRF-1 mediated apoptosis after treatment with ionizing radiation or chemotherapy drugs [38]. Overexpression of IRF-1 enhanced the chemosensitivity of gastric cancer cells to 5-fluorouracil [39], and high expression of IRF-1 also improved the efficiency of cisplatin-mediated killing of ovarian [40] and pancreatic cancer cells [41]. Therefore, we speculated that IRF-1 may disrupt mitochondrial homeostasis, causing mitochondrial depolarization and oxidative stress to promote apoptotic cell death in A549 cells.

Autophagy is a defense and adaptive response and cytoprotective mechanism that is important for the quality control process that maintains mitochondrial homeostasis [42]. Mitochondrial dysfunction or disruption of mitochondrial homeostasis, such as a respiratory injury or membrane 
A

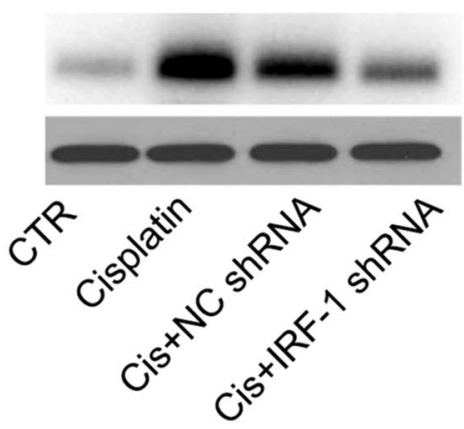

IRF-1 48KD

Histone H3 17KD
C

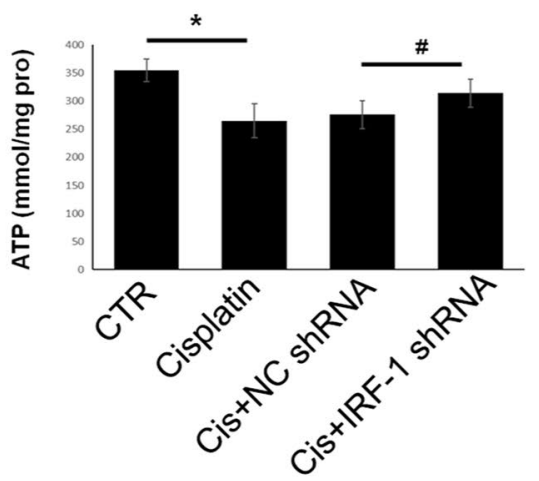

B

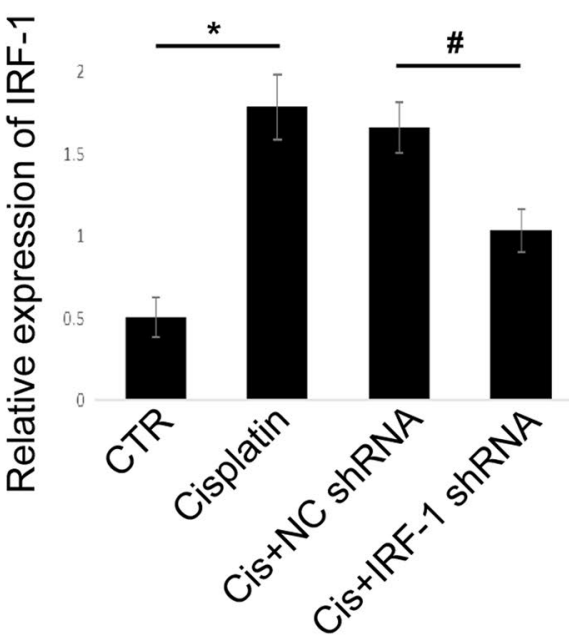

D

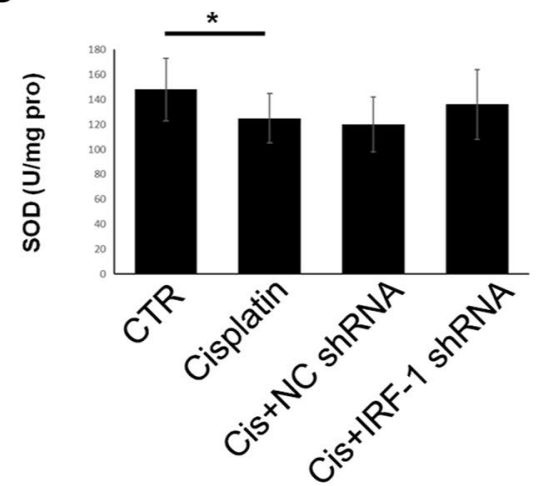

E

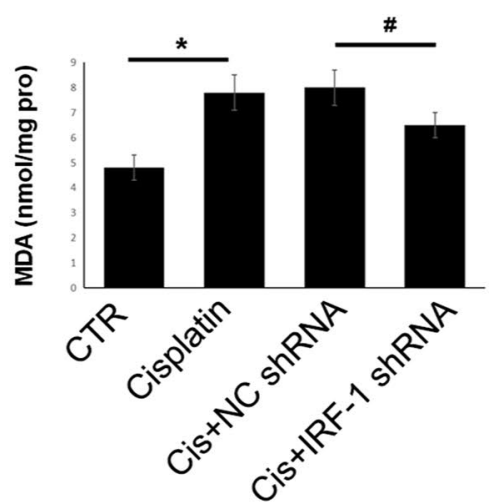

Fig. 6 Knockdown of IRF-1 protects against cisplatin-induced mitochondrial depolarization and oxidative stress. A, B Western blotting protein bands and corresponding quantitative analysis show the expression of IRF-1 in each group; the levels of ATP (C), SOD (D),

depolarization, and excessive ROS accumulation have been implicated in tumorigenesis and drug responsiveness in multiple tumors [43]. Autophagy improves the tolerance of tumor cells to unfavorable environments as well as their survival under cisplatin treatment [44], which is the mechanism driving cisplatin resistance; thus inhibition of autophagy may reverse cisplatin resistance. In lung cancer and other malignant tumors, it has been suggested that enhanced autophagy may be related to drug resistance $[45,46]$. In this study, we found that IRF-1 could inhibit autophagy, which might explain the reversal of cisplatin resistance in A549 cells.

This study preliminarily proposed that IRF-1 might be a possible target for increasing the sensitivity to cisplatin therapy by regulating apoptosis, autophagy, and mitochondrial homeostasis, and further studies were needed to elucidate the underlying exact mechanism. For example, cisplatin-induced apoptosis has been recognized as an important and MDA (E) as determined using respective test kits. $* P<0.05$ vs. the control, $\# P<0.05$ vs. NC shRNA. CTR, control; NC, negative control

mechanism for its anti-tumor activity. The pathways of apoptosis can be divided into endogenous mitochondrial pathway, endogenous endoplasmic reticulum pathway and exogenous death receptor pathway [47]. In terms of endogenous mitochondrial apoptotic pathway, when the mitochondrial membrane potential decreases, the mitochondrial membrane permeability increases, and the pro-apoptotic factors in the mitochondria are released into the cytoplasm. After cytochrome $\mathrm{C}$ is released from mitochondrial into the cell, it interacts with apoptotic protease activating factor-1 (APAF1) and forms apoptotic complex with the assistance of ATP and dATP, which forms caspase-9 holoenzyme by recruiting and activating pro-caspase-9. Caspase- 9 holoenzyme further activates effector caspase 3 and caspase-7, initiating the Caspase cascade, which ultimately leads to apoptosis [48-50]. In this study, we found that IRF-1 disrupted mitochondrial homeostasis, causing mitochondrial depolarization to promote apoptotic cell death in A549 cells, and therefore 

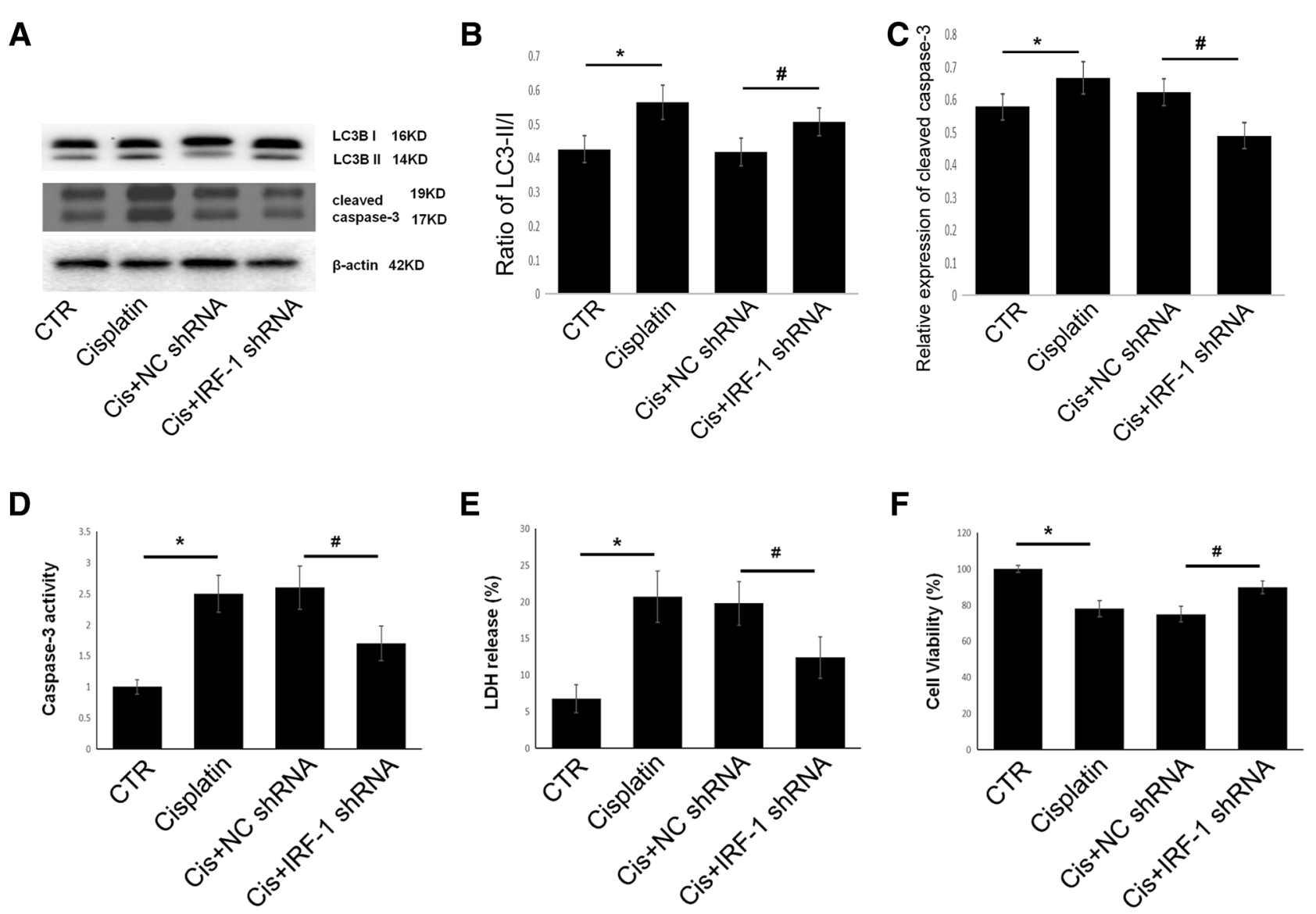

Fig. 7 Knockdown of IRF-1 reduces cisplatin-induced apoptosis and promotes autophagy. A-C Western blotting proteins bands and corresponding quantitative analysis show the expression of cleaved caspase 3 and LC3-I/II; D, caspase 3 activity as determined by caspase

we speculated that an endogenous mitochondrial apoptotic pathway was involved. However, only caspase 3 activity was detected, this speculation should be further confirmed by detecting other factors, such as caspase-9. Additionally, the regulatory mechanism of autophagy requires further study.

\section{Conclusions}

In conclusion, we found that IRF-1 is depleted in NSCLC, and cisplatin induces IRF-1 activation. IRF-1 overexpression promotes apoptotic cell death, inhibits autophagy, and induces mitochondrial depolarization and oxidative stress.
3 activity assay kit; $\mathbf{E}$ LDH as detected by LDH release reagent; $\mathbf{F}$ cell viability as determined by MTT assay. $* P<0.05$ vs. the control group, $\# P<0.05$ vs. NC shRNA. CTR, control; NC, negative control

Our study is helpful in understanding the mechanisms by which cisplatin kills lung cancer cells and provides further compelling evidence that IRF-1 is involved in the anti-cancer effect of cisplatin by promoting apoptosis and inhibiting autophagy to disturb mitochondrial homeostasis (Fig. 8). These results suggest IRF-1 as a possible target for increasing the sensitivity to cisplatin therapy by regulating apoptosis, autophagy, and mitochondrial homeostasis. Our data also provide novel mechanistic insights into IRF-1 as an effector of cisplatin and the possibility of enhancing chemotherapy sensitivity by upregulating IRF-1 expression.

Acknowledgements This study was supported by Grants from the National Natural Science Foundation of Hunan Province (2020JJ4418, 2020RC3067), Clinical Medical Technology Innovation Guided Project (2020SK51112), Natural Science Foundation of Hunan Province National Health Commission (B2019091, 20201286), Natural Science Foundation of Changsha Science and Technology Bureau (Kq1901084, Kq1901080, Kq2001024), and Cancer Foundation of China (NCC2018B58), Key Research and Development Project of 


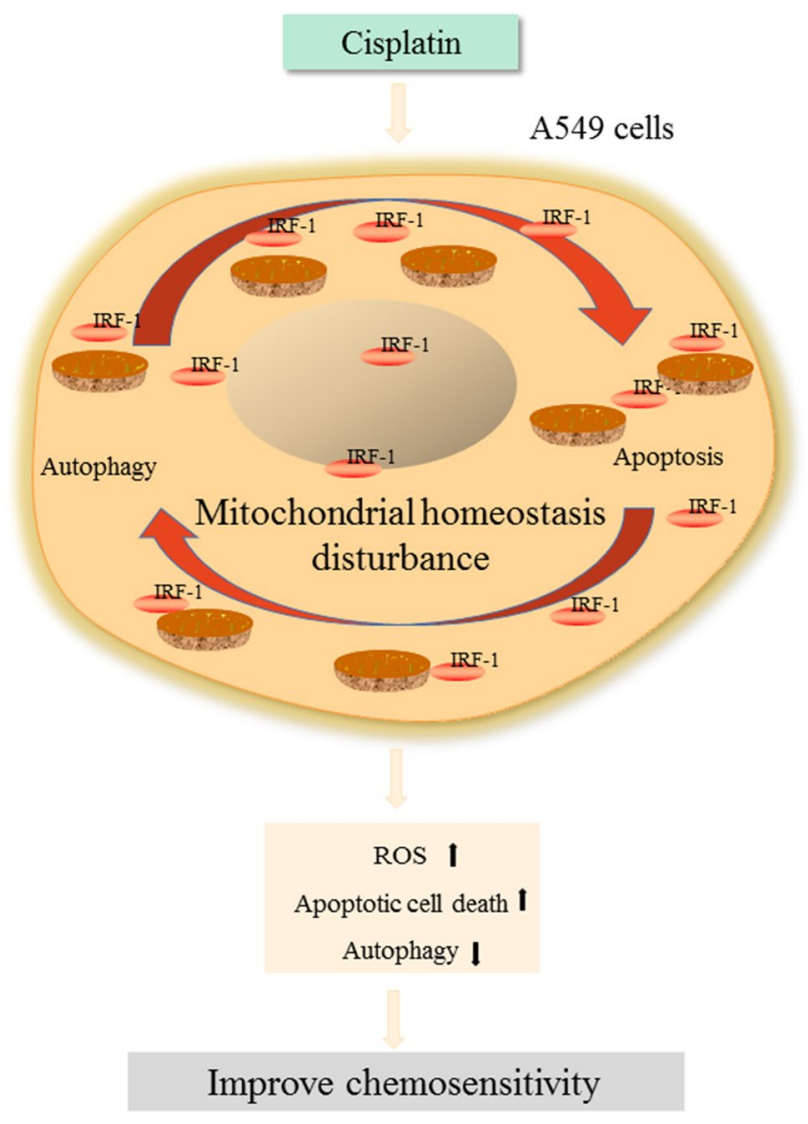

Fig. 8 Schematic showing the biologic role of IRF-1 in lung cancer

Hunan Province (2017WK2061); HUI LAN PUBLIC FOUNDATION (HL-HS2020-1).

Author contributions $\mathrm{LZ}$ and TC contribute to clinical data collection. HY, JC, XW, ZJ, HY contribute to molecular experiments and data statistics analyze. LZ and YL contribute to manuscript preparation. LZ and YL contribute to the study design, manuscript writing and data analyze. All authors read and approved the final manuscript.

Contribution to the field statement The expression of IRF-1 is decreased in NSCLC tumor tissues. Cisplatin induces IRF-1 activation, ROS production, and mitochondrial depolarization. IRF-1 overexpression promoted apoptotic cell death, inhibited autophagy, and induced oxidative stress; while IRF-1 knockdown reversed these effects. These data suggest that IRF-1 regulates apoptosis, autophagy and oxidative stress, which might be served as a potential target for increasing chemotherapy sensitivity of NSCLC.

Data availability The raw data, supporting the conclusions of this manuscript, will be made available by contacting the corresponding author.

\section{Declarations}

Conflict of interest The authors have declared that no competing interest exists.
Ethical approval The Ethics Committee of Hunan Cancer Hospital approved this study, and informed consent was obtained from all study patients.

Open Access This article is licensed under a Creative Commons Attribution 4.0 International License, which permits use, sharing, adaptation, distribution and reproduction in any medium or format, as long as you give appropriate credit to the original author(s) and the source, provide a link to the Creative Commons licence, and indicate if changes were made. The images or other third party material in this article are included in the article's Creative Commons licence, unless indicated otherwise in a credit line to the material. If material is not included in the article's Creative Commons licence and your intended use is not permitted by statutory regulation or exceeds the permitted use, you will need to obtain permission directly from the copyright holder. To view a copy of this licence, visit http://creativecommons.org/licenses/by/4.0/.

\section{References}

1. Yang D, Liu Y, Bai C, Wang X, Powell CA. Epidemiology of lung cancer and lung cancer screening programs in China and the United States. Cancer Lett. 2020;468:82-7.

2. Duma N, Santana-Davila R, Molina JR. Non-small cell lung cancer: epidemiology, screening, diagnosis, and treatment. Mayo Clin Proc. 2019;94(8):1623-40.

3. Sapalidis K, Zarogoulidis P, Huang H, Bai C, Wen Y, Wang L, et al. Inhaled immunotherapy administration for lung cancer; efficient? Certainly possible. J Cancer. 2018;9(6):1121-6.

4. Uras IZMH, Casanova E. Targeting KRAS mutant nonsmall-cell lung cancer: past, present and future. Int J Mol Sci. 2020;21(12):4325.

5. Chen Q, Guo Z, Liu S, Quan P, Cao X, Guo L, et al. The cancer incidence and mortality among children and adolescents during the period of 2010-2014 in Henan Province, China. Cancer Med. 2019;8(2):814-23.

6. Griesinger F, Korol EE, Kayaniyil S, Varol N, Ebner T, Goring SM. Efficacy and safety of first-line carboplatin-versus cisplatinbased chemotherapy for non-small cell lung cancer: a meta-analysis. Lung Cancer. 2019;135:196-204.

7. Li X, Guo S, Xiong XK, Peng BY, Huang JM, Chen MF, et al. Combination of quercetin and cisplatin enhances apoptosis in OSCC cells by downregulating xIAP through the NF-кB pathway. J Cancer. 2019;10(19):4509-21.

8. Zhu J, Zou H, Yu W, Huang Y, Liu B, Li T, et al. Checkpoint kinase inhibitor AZD7762 enhance cisplatin-induced apoptosis in osteosarcoma cells. Cancer Cell Int. 2019;19:195.

9. Rada M, Nallanthighal S, Cha J, Ryan K, Sage J, Eldred C, et al. Inhibitor of apoptosis proteins (IAPs) mediate collagen type XI alpha 1-driven cisplatin resistance in ovarian cancer. Oncogene. 2018;37(35):4809-20.

10. Wang H, Chen J, Zhang S, Zheng X, Xie S, Mao J, et al. MiR-223 regulates autophagy associated with cisplatin resistance by targeting FBXW7 in human non-small cell lung cancer. Cancer Cell Int. 2020;20:258.

11. Zhou F, Yang X, Zhao H, Liu Y, Feng Y, An R, et al. Downregulation of OGT promotes cisplatin resistance by inducing autophagy in ovarian cancer. Theranostics. 2018;8(19):5200-12.

12. Gillissen B, Richter A, Richter A, Preissner R, Schulze-Osthoff K, Essmann F, et al. Bax/Bak-independent mitochondrial depolarization and reactive oxygen species induction by sorafenib overcome resistance to apoptosis in renal cell carcinoma. J Biol Chem. 2017;292(16):6478-92. 
13. Han Y, Kim B, Cho U, Park IS, Kim SI. Mitochondrial fission causes cisplatin resistance under hypoxic conditions via ROS in ovarian cancer cells. Oncogene. 2019;38(45):7089-105.

14. Deng SY, Zhang LM, Ai YH, Pan PH, Zhao SP, Su XL, et al. Role of interferon regulatory factor-1 in lipopolysaccharide-induced mitochondrial damage and oxidative stress responses in macrophages. Int J Mol Med. 2017;40(4):1261-9.

15. Feng HZY, Gui JF, Lemon SM, Yamane D. Interferon regulatory factor 1 (IRF1) and anti-pathogen innate immune responses. PLoS Pathog. 2021;17(1):e1009220.

16. Khan T, Ganai BA, Masood A, Samoon J, Beigh SR, Qazi F. Relation between IRF-1 gene and acute myelocytic leukemia in Kashmiri population. Asian Pac J Cancer Prev. 2011;12(4):1035-9.

17. Wang B, Yang H, Shen L, Wang J, Pu W, Chen Z, et al. Rs56288038 (C/G) in 3'UTR of IRF-1 regulated by MiR-502-5p promotes gastric cancer development. Cell Physiol Biochem. 2016;40(1-2):391-9.

18. Sers CHK, Nazarenko I, Reich S, Wiechen K, Zhumabayeva B, Adhikari P, Schröder K, Gontarewicz A, Schäfer R. The class II tumour suppressor gene H-REV107-1 is a target of interferonregulatory factor- 1 and is involved in IFN $\gamma$-induced cell death in human ovarian carcinoma cells. Oncogene. 2002;21(18):2829-39.

19. Bowie MLIC, Seewalt VL. IRF-1 promotes apoptosis in p53-damaged basal-type human mammary epithelial cells: a model for early basal-type mammary carcinogenesis. Adv Exp Med Biol. 2008;617:367-74.

20. Cook KL, Schwartz-Roberts JL, Baumann WT, Clarke R. Linking autophagy with inflammation through IRF1 signaling in ER+ breast cancer. Mol Cell Oncol. 2016;3(1):e1023928.

21. Schwartz-Roberts JL, Cook KL, Chen C, Shajahan-Haq AN, Axelrod M, Wrri A, et al. Interferon regulatory factor-1 signaling regulates the switch between autophagy and apoptosis to determine breast cancer cell fate. Cancer Res. 2015;75(6):1046-55.

22. Zhang L, Cardinal JS, Bahar R, Evankovich J, Huang H, Nace $\mathrm{G}$, et al. Interferon regulatory factor-1 regulates the autophagic response in LPS-stimulated macrophages through nitric oxide. Mol Med. 2012;18(1):201-8.

23. Yuan J, Yin Z, Tan L, Zhu W, Tao K, Wang G, et al. Interferon regulatory factor-1 reverses chemoresistance by downregulating the expression of P-glycoprotein in gastric cancer. Cancer Lett. 2019;457:28-39.

24. Tan L, Yuan J, Zhu W, Tao K, Wang G, Gao J. Interferon regulatory factor-1 suppresses DNA damage response and reverses chemotherapy resistance by downregulating the expression of RAD51 in gastric cancer. Am J Cancer Res. 2020;10(4):1255-70.

25. Tang Z, Li C, Kang B, Gao G, Li C, Zhang Z. GEPIA: a web server for cancer and normal gene expression profiling and interactive analyses. Nucleic Acids Res. 2017;45(W1):W98-w102.

26. Haeussler M, Zweig AS, Tyner C, Speir ML, Rosenbloom KR, Raney BJ, et al. The UCSC genome browser database: 2019 update. Nucleic Acids Res. 2019;47(D1):D853-8.

27. Thul PJ, Lindskog C. The human protein atlas: a spatial map of the human proteome. Protein Sci. 2018;27(1):233-44.

28. Du Y, Zhang W, Du B, Zang S, Wang X, Mao X, et al. TRIM32 overexpression improves chemoresistance through regulation of mitochondrial function in non-small-cell lung cancers. Onco Targets Ther. 2018;11:7841-52.

29. Chen J, Zhang L, Zhou H, Wang W, Luo Y, Yang H, et al. Inhibition of autophagy promotes cisplatin-induced apoptotic cell death through Atg5 and Beclin 1 in A549 human lung cancer cells. Mol Med Rep. 2018;17(5):6859-65.

30. Armstrong MJ, Stang MT, Liu Y, Yan J, Pizzoferrato E, Yim JH. IRF-1 inhibits NF-kappaB activity, suppresses TRAF2 and cIAP1 and induces breast cancer cell specific growth inhibition. Cancer Biol Ther. 2015;16(7):1029-41.
31. Yan Y, Zheng L, Du Q, Yan B, Geller DA. Interferon regulatory factor 1 (IRF-1) and IRF-2 regulate PD-L1 expression in hepatocellular carcinoma (HCC) cells. Cancer Immunol Immunother. 2020;69(9):1891-903.

32. Pavan $\mathrm{S}$, Olivero $\mathrm{M}$, Corà $\mathrm{D}$, Di Renzo MF. IRF-1 expression is induced by cisplatin in ovarian cancer cells and limits drug effectiveness. Eur J Cancer. 2013;49(4):964-73.

33. de Oliveira MRNS, Manayi A, Daglia M, Hajheydari Z, Nabavi SM. Resveratrol and the mitochondria: from triggering the intrinsic apoptotic pathway to inducing mitochondrial biogenesis, a mechanistic view. Biochim Biophys Acta. 2016;1860(4):727-45.

34. Kang Z, Qiao N, Liu G, Chen H, Tang Z, Li Y. Copper-induced apoptosis and autophagy through oxidative stress-mediated mitochondrial dysfunction in male germ cells. Toxicol In Vitro: Int J Publ Assoc BIBRA. 2019;61:104639.

35. Xie LL, Shi F, Tan Z, Li Y, Bode AM, Cao Y. Mitochondrial network structure homeostasis and cell death. Cancer Sci. 2018;109(12):3686-94.

36. Pariente R, Pariente JA, Rodríguez AB, Espino J. Melatonin sensitizes human cervical cancer HeLa cells to cisplatin-induced cytotoxicity and apoptosis: effects on oxidative stress and DNA fragmentation. J Pineal Res. 2016;60(1):55-64.

37. Jeong SI, Kim JW, Ko KP, Ryu BK, Lee MG, Kim HJ, et al. XAF1 forms a positive feedback loop with IRF-1 to drive apoptotic stress response and suppress tumorigenesis. Cell Death Dis. 2018;9(8):806.

38. Jiang GM, Wang HS, Du J, Ma WF, Wang H, Qiu Y, et al. Bortezomib relieves immune tolerance in nasopharyngeal carcinoma via STAT1 suppression and indoleamine 2,3-dioxygenase downregulation. Cancer Immunol Res. 2017;5(1):42-51.

39. Gao J, Tian Y, Zhang J. Overexpression of interferon regulatory factor 1 enhances chemosensitivity to 5-fluorouracil in gastric cancer cells. J Cancer Res Ther. 2012;8(1):57-61.

40. Pavan SOM, Corà D, Di Renzo MF. IRF-1 expression is induced by cisplatin in ovarian cancer cells and limits drug effectiveness. Eur J Cancer. 2013;49(4):964-73.

41. Mashima $\mathrm{H}$, Sakai $\mathrm{T}$, Ohnishi $\mathrm{H}$. The roles of interferon regulatory factors in pancreatic diseases. Suizo. 2014;29(1):23-31.

42. Wang R, Wang G. Autophagy in mitochondrial quality control. Adv Exp Med Biol. 2019;1206:421-34.

43. Guerra F, Arbini AA, Moro L. Mitochondria and cancer chemoresistance. Biochim Biophys Acta Bioenerg. 2017;1858(8):686-99.

44. Li YJ, Lei YH, Yao N, Wang CR, Hu N, Ye WC, et al. Autophagy and multidrug resistance in cancer. Chin J Cancer. 2017;36(1):52.

45. Ma K, Li S, Huo X, Guo M, Du X, Li C, et al. Exploring the mechanism of cisplatin resistance by transcriptome sequencing and reversing the chemoresistance by autophagy inhibition in small cell lung cancer. Biochem Biophys Res Commun. 2020;533(3):474-80.

46. Li N, Li X, Li S, Zhou S, Zhou Q. Cisplatin-induced downregulation of SOX1 increases drug resistance by activating autophagy in non-small cell lung cancer cell. Biochem Biophys Res Commun. 2013;439(2):187-90.

47. Danial NN, Korsmeyer SJ. Cell death: critical control points. Cell. 2004;116(2):205-19.

48. Chalah A, Khosravi-Far R. The mitochondrial death pathway. Adv Exp Med Biol. 2008;615:25-45.

49. Pradelli LA, Bénéteau M, Ricci JE. Mitochondrial control of caspase-dependent and -independent cell death. Cell Mol Life Sci. 2010;67(10):1589-97.

50. Zhang TM. TRIAP1 inhibition activates the cytochrome c/Apaf-1/ Caspase-9 signaling pathway to enhance human ovarian cancer sensitivity to cisplatin. Chemotherapy. 2019;64(3):119-28.

Publisher's Note Springer Nature remains neutral with regard to jurisdictional claims in published maps and institutional affiliations. 